\title{
A Class of Population Dynamics for Reaching Epsilon-Equilibria: Engineering Applications
}

\author{
Germán Obando, Julian Barreiro-Gomez, and Nicanor Quijano
}

\begin{abstract}
This document proposes a novel class of population dynamics that are parameterized by a nonnegative scalar $\epsilon$. We show that any rest point of the proposed dynamics corresponds to an $\epsilon$-equilibrium of the underlying population game. In order to derive this class of population dynamics, our approach is twofold. First, we use an extension of the pairwise comparison revision protocol and the classic mean dynamics for well-mixed populations. This approach requires full-information. Second, we employ the same revision protocol and a version of the mean dynamics for non-well-mixed populations that uses only local information. Furthermore, invariance properties of the set of allowed population states are analyzed, and stability of the $\epsilon$-equilibria is formally proven. Finally, two engineering examples based on the $\epsilon$-dynamics are presented: A control scenario in which noisy measurements should be mitigated, and a humanitarian engineering application related to wealth distribution in poor societies.
\end{abstract}

\section{INTRODUCTION}

In game theory, the $\epsilon$-equilibrium is a relaxation of the widely known concept of Nash equilibrium [1]. While in a Nash equilibrium a player has no incentives to unilaterally change its strategy, in an $\epsilon$-equilibrium players can have incentives to choose other strategies. Nonetheless, these incentives are not higher than a nonnegative threshold $\epsilon$. There are a couple of issues that motivate the development of techniques to find a relaxed version of Nash equilibria. First, $\epsilon$-equilibrium is used in stochastic games or games with imperfect information, where Nash equilibrium is rapidly changing. Under this class of games, it can be desirable that players' decisions converge to a rest point that is close to the Nash equilibrium instead of having permanent variations of the players' decisions due to Nash equilibrium changes. Second, there are some cases in which finding a Nash equilibrium is computationally expensive [2]. In these cases, convergence to $\epsilon$-equilibria reduces the computational burden. Although algorithms for reaching $\epsilon$-equilibria have been proposed for strategic games (e.g., see [3]), similar methods have been largely unexplored for population games.

G. Obando, J. Barreiro-Gomez, and N. Quijano are with Departamento de Ingeniería Eléctrica y Electrónica, Universidad de los Andes, Carrera 1 No 18A-10, Bogotá, Colombia \{ge-oband, j.barreiro135, nquijano\}@uniandes .edu.co.

J. Barreiro-Gomez is also with the Automatic Control Department, Universitat Politècnica de Catalunya, Institut de Robòtica i Informàtica Industrial (CSIC-UPC), Llorens i Artigas, 4-6, 08028 Barcelona, Spain; and with the project ECOCIS (Ref. DPI2013-48243-C2-1-R) from the Spanish Ministry of Science and Education.

This work was supported in part by Colciencias Colfuturo, under Grants 528 and 6172 , and in part by the project ALTERNAR, BPIN 20130001000089, OCAD-Fondo de CTel-SGR-Colombia, under Grant Acuerdo 005 de 2013. Agència de Gestió d'Ajust Universitaris i de Recerca AGAUR also supports J. Barreiro-Gomez.
The contribution of this work is the deduction of a class of population dynamics that are parameterized by a nonnegative escalar $\epsilon$ and converge to the set of $\epsilon$-equilibria of the underlying game. Indeed, we formally show that the set of epsilon-equilibria and the rest points of the proposed $\epsilon$ dynamics are equal. We use two approaches to derive the $\epsilon$-dynamics. On the one hand, we employ a parameterized version of the pairwise comparison protocol and the mean dynamics for well-mixed populations [4]. This approach generates a version of the $\epsilon$-dynamics that requires fullinformation of the game to evolve. On the other hand, we use the same modified pairwise comparison protocol and the mean dynamics for non-well-mixed populations [5] to obtain distributed $\epsilon$-dynamics that only use local information.

Due to the advantages of finding $\epsilon$-equilibria instead of Nash equilibria, $\epsilon$-dynamics enlarge the spectrum of applications of population games, which have been widely employed for solving optimization and control problems [6], [7]. For instance, since a controller based on $\epsilon$-dynamics can deal with imperfect information, it is suitable to be applied in situations where the measurements of systems' outputs have noise. To illustrate the usefulness of $\epsilon$-dynamics, we apply them in two problems: i) The design of a modelfree controller for water allocation in an $m$-tanks system that includes noisy measurements; ii) the design of wealth distribution policies in poor communities. The latter problem is discussed in [8].

The organization of this document is as follows: Section II presents the preliminary concepts associated with population dynamics, $\epsilon$-equilibria, and graphs. Section III introduces the deduction of the $\epsilon$-dynamics. Afterwards, some properties of the $\epsilon$-dynamics are presented in IV. Section V derives the distributed version of the $\epsilon$-dynamics. Moreover, Sections VI and VII present applications of the $\epsilon$-dynamics in a control problem with noisy measurements, and the formulation of a wealth distribution policy, respectively. Finally, Section VIII draws come concluding remarks.

Notation: Throughout this manuscript, we use $\mathbb{R}_{+}^{n}$ to denote the set of vectors in $\mathbb{R}^{n}$ that have nonnegative entries. Similarly, $\mathbb{R}_{++}^{n}$ denotes the set of vectors in $\mathbb{R}^{n}$ with strictly positive entries. On the other hand, we use $[\cdot]_{+}$to denote the projection on $\mathbb{R}_{++}$, i.e., $[\cdot]_{+}=\max (0, \cdot)$.

\section{PRELIMINARIES}

\section{A. Population Games}

A population game is a model that describes the strategic interaction among individuals in a population. Mathematically, a population game is characterized by: a population 
mass $X \in \mathbb{R}_{+}$; a set of strategies $\mathcal{S}=\{1, \ldots, n\}$; a population state $x=\left[x_{1}, \ldots, x_{n}\right]^{\top}$ that defines the portion of the population mass playing each strategy, i.e., $x \in \Delta$, where

$$
\Delta=\left\{x \in \mathbb{R}_{+}^{n}: \sum_{i=1}^{n} x_{i}=X\right\}
$$

and a set of fitness functions $f_{i}: \Delta \mapsto \mathbb{R}$, for all $i=$ $1, \ldots, n$. Given a population state $x$, the fitness value $f_{i}(x)$ captures the payoff of the individuals playing the $i^{\text {th }}$ strategy. For notational convenience, we define the fitness vector $f(x):=\left[f_{1}(x), \ldots, f_{n}(x)\right]^{\top}$.

Under the assumption that the individuals of the population are rational, i.e., they try to maximize their payoff, the expected output of a population game is given by a Nash equilibrium.

Definition 1: Nash equilibria (adapted from [4]). $x^{*} \in$ $\Delta$ is a Nash equilibrium if each used strategy entails the maximum benefit for the players who chose it. Equivalently, the set of Nash equilibria is given by $N E=\left\{x^{*} \in \Delta\right.$ : $\left.x_{i}^{*}>0 \Rightarrow f_{i}\left(x^{*}\right) \geq f_{j}\left(x^{*}\right), \forall i, j=1, \ldots, n\right\}$.

A relaxed version of the Nash equilibirum concept is the $\epsilon$-equilibrium [1], which is defined as follows:

Definition 2: $\epsilon$-equilibria. Let $\epsilon \in \mathbb{R}_{+}$. The set of $\epsilon$ equilibria is given by $E_{\epsilon}=\left\{x^{\epsilon} \in \Delta: x_{i}^{\epsilon}>0 \Rightarrow f_{i}\left(x^{\epsilon}\right) \geq\right.$ $\left.f_{j}\left(x^{\epsilon}\right)-\epsilon, \forall i, j=1, \ldots, n\right\}$.

While in a Nash equilibrium an individual has no incentives to unilaterally change its strategy, in an $\epsilon$-equilibrium the individuals can have incentives to choose other strategies, but these incentives are not higher than a threshold $\epsilon$. Notice that, if $\epsilon=0$, an $\epsilon$-equilibrium is a Nash equilibrium.

\section{B. Graphs}

Using the framework developed in [5], the structure of a population involved in a strategic game can be modeled by means of a graph $\mathcal{G}=\{\mathcal{V}, \mathcal{E}\}$, where the set of nodes $\mathcal{V}$ is associated with the available strategies, i.e., $\mathcal{V}=\mathcal{S}$, and the set of edges $\mathcal{E} \subset \mathcal{V} \times \mathcal{V}$ describes the encounter probability between individuals playing different strategies, i.e., if $(i, j) \in \mathcal{E}$, then it is possible that an individual playing strategy $i$ can be matched with an individual playing strategy $j$. In contrast, if $(i, j) \notin \mathcal{E}$, then individuals playing strategies $i$ and $j$ cannot encounter each other. Throughout this paper, we only consider undirected graphs, i.e., if $(i, j) \in \mathcal{E}$, then $(j, i) \in \mathcal{E}$.

A well-mixed population is such that the encounter probability of individuals playing any two strategies from $\mathcal{S}$ is the same no matter what the strategies are. Therefore, wellmixed population are represented by complete graphs. On the other hand, in non-well-mixed populations, the encounter probability between individuals depends on the strategies that these individuals are playing. Hence, non-well-mixed populations are described by non-complete graphs. In [5], non-well-mixed populations are employed to model local information structures.

\section{EPSILON-DYNAMICS}

\section{A. Revision Protocols and Mean Dynamics}

Nash equilibria and $\epsilon$-equilibria predict the outcome of a population game. Different from these static notions, there exists a framework that is capable to model not only the outcome of a population game, but also the evolution of the population state along the time. This framework is called population dynamics, and its fundamental input is the concept of revision protocol.

A revision protocol is a set of rules that describes the timing and results of the individuals decisions. Specifically, a revision protocol establishes conditions under which an individual changes its strategy. Formally, a revision protocol is defined as follows,

Definition 3: Revision protocol (adapted from [4]). A revision protocol, denoted by $\rho=\left[\rho_{i j}\right]$, is a map $\rho$ : $\mathbb{R}^{n} \times \Delta \mapsto \mathbb{R}_{+}^{n \times n}$ that is characterized by the conditional switch rates $\left\{\rho_{i j}: i, j \in \mathcal{S}\right\}$. Given a population state $x$ and a fitness vector $f(x), \rho_{i j}(f(x), x)$ captures the switch rate from the $i^{\text {th }}$ strategy to the $j^{\text {th }}$ strategy.

A population game and a revision protocol define the following dynamics,

$$
\dot{x}_{i}=\sum_{j \in \mathcal{S}} x_{j} \rho_{j i}(f(x), x)-x_{i} \sum_{j \in \mathcal{S}} \rho_{i j}(f(x), x), \forall i \in \mathcal{S},
$$

which are called mean dynamics [4]. The first term of (2) is related to the individuals that switch to the $i^{\text {th }}$ strategy from other ones, while the second term is associated with the individuals that change strategy $i \in \mathcal{S}$ by another strategy.

Summarizing, (2) models the dynamics of a population involved in a strategic game that is using a given revision protocol. In this regard, different revision protocols produce different population dynamics models.

\section{B. Epsilon-Dynamics}

Let us define the following revision protocol,

$$
\rho_{i j}(f(x), x)=\left[f_{j}(x)-\left(f_{i}(x)+\epsilon\right)\right]_{+}, \forall i, j \in \mathcal{S},
$$

where $\epsilon \in \mathbb{R}_{+}$. The constant $\epsilon$ acts as a switching threshold. That is, an individual playing the $i^{t h}$ strategy that adopts the revision protocol (3) might prefer to keep strategy $i$ even if strategy $j$ entails a higher payoff. This individual only switches to the $j^{\text {th }}$ strategy if the difference between the payment perceived by using the $j^{\text {th }}$ strategy and the payment perceived by using its current strategy exceeds the value of $\epsilon$. Individuals' self-confidence, change aversion, opinion inertia, and other kind of biases can be modeled by protocol (3).

Replacing the revision protocol (3) in the mean dynamics (2), we obtain the proposed $\epsilon$-dynamics,

$$
\begin{aligned}
\dot{x}_{i}= & \sum_{j \in \mathcal{S}} x_{j}\left[f_{i}(x)-\left(f_{j}(x)+\epsilon\right)\right]_{+} \\
& -x_{i} \sum_{j \in \mathcal{S}}\left[f_{j}(x)-\left(f_{i}(x)+\epsilon\right)\right]_{+}, \forall i \in \mathcal{S} .
\end{aligned}
$$


Notice that, if $\epsilon=0$, the revision protocol (3) becomes a pairwise comparison protocol [4], and the $\epsilon$-dynamics become the classic Smith dynamics. Nevertheless, the addition of a parameter $\epsilon>0$ provides different characteristics to the behavior of a population that evolves according to the $\epsilon$-dynamics compared to a population under the Smith dynamics. These characteristics are studied in the next section.

\section{EPSILON-DyNAMICS PROPERTIES}

\section{A. Simplex Invariance}

First, let us show that the $\epsilon$-dynamics satisfy simplex invariance, which is a fundamental property of population dynamics. The invariance of the simplex $\Delta$, defined in (1), guarantees that the population mass remains constant, and that the population state $x$ belongs to $\mathbb{R}_{+}^{n}$ for all time, i.e., the amount of individuals playing any strategy is always nonnegative.

Proposition 1: Simplex invariance. Assume that the initial population state $x(0) \in \Delta$. If $x(t)$ evolves according to the $\epsilon$-dynamics (4), then $x(t) \in \Delta$, for all $t \geq 0$.

Proof: Notice that $x(t) \in \Delta$ if $x(t)$ satisfies two conditions (cf. Equation (1)): i) mass conservation, i.e., $\sum_{i \in \mathcal{S}} x_{i}(t)=X$; and $\left.i i\right)$ nonnegativeness, i.e., $x_{i}(t) \geq 0$, $\forall i \in \mathcal{S}$. Therefore, our proof is divided into two steps:

1. Mass conservation: Since $\sum_{i \in \mathcal{S}} x_{i}(0)=X$ by assumption, to prove mass conservation it is sufficient to show that the quantity $\sum_{i \in \mathcal{S}} x_{i}(t)$ is positively invariant. Notice that $\sum_{i \in \mathcal{S}} \dot{x}_{i}=\sum_{j \in \mathcal{S}} x_{j} \sum_{i \in \mathcal{S}}\left[f_{i}(x)-\left(f_{j}(x)+\epsilon\right)\right]_{+}-$ $\sum_{i \in \mathcal{S}} x_{i} \sum_{j \in \mathcal{S}}\left[f_{j}(x)-\left(f_{i}(x)+\epsilon\right)\right]_{+}$. Rewriting the right-hand side of this mathematical equation, we obtain that $\sum_{i \in \mathcal{S}} \dot{x}_{i}=\sum_{j \in \mathcal{S}} x_{j} \sum_{i \in \mathcal{S}}\left[f_{i}(x)-\left(f_{j}(x)+\epsilon\right)\right]_{+}-$ $\sum_{j \in \mathcal{S}} x_{j} \sum_{i \in \mathcal{S}}\left[f_{i}(x)-\left(f_{j}(x)+\epsilon\right)\right]_{+}$. Thus, $\sum_{i \in \mathcal{S}} \dot{x}_{i}=0$. This implies that $\sum_{i \in \mathcal{S}} x_{i}(t)$ is positively invariant. Hence, $\sum_{i \in \mathcal{S}} x_{i}(t)=X$, for all $t \geq 0$.

2. Nonnegativeness: Let us prove that $x_{i}(t) \geq 0$, for all $i \in \mathcal{S}$, and for all $t \geq 0$. We proceed by contradiction.

Since the initial state $x(0)$ belongs to $\mathbb{R}_{+}^{n}$, let us assume that there exists a set $\mathcal{S}^{\prime} \subseteq \mathcal{S}$ associated with the first state variables that become negative, i.e., we assume that, for some $t_{a}>0$, the following conditions hold: $i$ ) $\left\{x_{k}\left(t_{a}\right)<0: k \in \mathcal{S}^{\prime}\right\}$; and ii) $\left\{x_{k}(t) \geq 0: k \in \mathcal{S} \backslash \mathcal{S}^{\prime}\right\}$, for all $t<t_{a}$.

Notice that the above assumption implies that there exist $t_{b}<t_{a}$ and $l \in \mathcal{S}^{\prime}$ such that $x_{l}\left(t_{b}\right)=0, \dot{x}_{l}\left(t_{b}\right)<$ 0 , and $x_{j}\left(t_{b}\right) \geq 0$, for all $j \in \mathcal{S}$. This is a contradiction since, according to the $\epsilon$-dynamics $(4), \dot{x}_{l}\left(t_{b}\right)=$ $\sum_{j \in \mathcal{S}} x_{j}\left(t_{b}\right)\left[f_{l}\left(x\left(t_{b}\right)\right)-\left(f_{j}\left(x\left(t_{b}\right)\right)+\epsilon\right)\right]_{+} \geq 0$.

If the population mass is associated with a physical quantity (e.g., if the $\epsilon$-dynamics are used to model an engineering problem), then simplex invariance plays a fundamental role for guaranteeing the satisfaction of certain constraints on that quantity. For instance, if the population mass is associated with the energy applied by a control system, simplex invariance guarantees the limitation of that energy (see, e.g., [9], [10], [7], [11]). Benefits of simplex invariance are exploited in some applications that are discussed later in this paper.

\section{B. Convergence to Epsilon-Equilibria}

Depending on the revision protocol, population dynamics converge to rational outputs of games. In fact, several classic population dynamics, including best response, Smith, and projection dynamics converge to Nash equilibria. In this section, we show that the proposed $\epsilon$-dynamics converge to $\epsilon$-equilibria.

First, let us prove that there is an equivalence between rest points of the $\epsilon$-dynamics and $\epsilon$-equilibria of the underlying population game.

Proposition 2: Rest points. Let $\epsilon \in \mathbb{R}_{+}$. The population state $x^{\epsilon} \in \Delta$ is an equilibrium point of the $\epsilon$-dynamics (4) if and only if $x^{\epsilon} \in E_{\epsilon}$.

Proof:

- " $\Rightarrow$ ": Let $\hat{\mathcal{S}} \subseteq \mathcal{S}$ be the set of strategies that are used at equilibrium, i.e., $\hat{\mathcal{S}}=\left\{i \in \mathcal{S}: x_{i}^{\epsilon}>0\right\}$; and let $\hat{\mathcal{S}}^{\prime} \subseteq \mathcal{S}$ be the set of strategies that are not used at equilibrium, i.e., $\hat{\mathcal{S}}^{\prime}=\left\{i \in \mathcal{S}: x_{i}^{\epsilon}=0\right\}$. Notice that, since $x^{\epsilon} \in \Delta$ by assumption, then $\hat{\mathcal{S}} \cup \hat{\mathcal{S}}^{\prime}=\mathcal{S}$.

First, let us investigate the characteristics of the state variables related to $\hat{\mathcal{S}}^{\prime}$. According to (4), we have that $\sum_{j \in \hat{\mathcal{S}}} x_{j}^{\epsilon}\left[f_{i}\left(x^{\epsilon}\right)-\left(f_{j}\left(x^{\epsilon}\right)+\epsilon\right)\right]_{+}=0, \forall i \in \hat{\mathcal{S}}^{\prime}$. Thus,

$$
f_{i}\left(x^{\epsilon}\right) \leq f_{j}\left(x^{\epsilon}\right)+\epsilon, \forall i \in \hat{\mathcal{S}}^{\prime} \text { and } j \in \hat{\mathcal{S}} .
$$

Now, let us characterize the state variables related to $\hat{\mathcal{S}}$. Without loss of generality, assume that $\hat{\mathcal{S}}=\{n, n-$ $1, \ldots, k+1, k\}$, and $f_{n}\left(x^{\epsilon}\right) \geq f_{n-1}\left(x^{\epsilon}\right) \geq \cdots \geq$ $f_{k+1}\left(x^{\epsilon}\right) \geq f_{k}\left(x^{\epsilon}\right)$. Once this is made, we proceed by contradiction. Assume that $f_{n}\left(x^{\epsilon}\right)>f_{k}\left(x^{\epsilon}\right)+\epsilon$. Under this scenario, we can use the property stated in (5) to obtain $\sum_{j \in \mathcal{S}} x_{j}^{\epsilon}\left[f_{n}\left(x^{\epsilon}\right)-\left(f_{j}\left(x^{\epsilon}\right)+\epsilon\right)\right]_{+}>0$, and $x_{n} \sum_{j \in \mathcal{S}}\left[f_{j}\left(x^{\epsilon}\right)-\left(f_{n}\left(x^{\epsilon}\right)+\epsilon\right)\right]_{+}=0$. Notice that this implies that the right hand side of (4) is different from zero, which is a contradiction since $x^{\epsilon}$ is a rest point by assumption. Therefore, $f_{k}\left(x^{\epsilon}\right)+\epsilon \geq f_{n}\left(x^{\epsilon}\right)$. A consequence of this fact is that

$$
\max _{i \in \hat{\mathcal{S}}}\left(f_{i}\right)-\min _{i \in \hat{\mathcal{S}}}\left(f_{i}\right) \leq \epsilon .
$$

Combining (5) and (6) we can conclude that $x^{\epsilon} \in E_{\epsilon}$.

- " $\Leftarrow$ ": Since $x^{\epsilon} \in E_{\epsilon}$, then $f_{i}\left(x^{\epsilon}\right)-\epsilon \leq$ $f_{j}\left(x^{\epsilon}\right), \forall i, j \in \mathcal{S}$ such that $x_{j}^{\epsilon}>0$. Thus, $\sum_{j \in \mathcal{S}} x_{j}^{\epsilon}\left[f_{i}\left(x^{\epsilon}\right)-\left(f_{j}\left(x^{\epsilon}\right)+\epsilon\right)\right]_{+}=0, \forall i \in \mathcal{S}$. Moreover, notice that $x_{i}^{\epsilon} \sum_{j \in \mathcal{S}}\left[f_{j}\left(x^{\epsilon}\right)-\left(f_{i}\left(x^{\epsilon}\right)+\epsilon\right)\right]_{+}=$ $0, \forall i \in \mathcal{S}$. This equality holds since, if $x_{i}^{\epsilon}>0$, then $f_{i}\left(x^{\epsilon}\right) \geq f_{j}\left(x^{\epsilon}\right)-\epsilon$. Therefore, the right hand side of (4) is equal to zero. This implies that $x^{\epsilon}$ is a rest point of the $\epsilon$-dynamics.

Having shown that any equilibrium point of the $\epsilon$ dynamics is an $\epsilon$-equilibrium, let us make some assumptions on the characteristics of the population game that guarantee that the $\epsilon$-dynamics converge to the set of $\epsilon$-equilibria.

Assumption 1: The fitness functions satisfy the following conditions, for all $i \in \mathcal{S}$, and $x \in \Delta$ : 
- $f_{i}$ only depends on the population playing the $i^{\text {th }}$ strategy, i.e., $x_{i}$ (when this is the case, we write $f_{i}\left(x_{i}\right)$ to make the dependency explicit).

- $f_{i}$ is differentiable. Moreover, its derivative satisfies $\frac{d f_{i}}{d x_{i}}\left(x_{i}\right)<0$.

Assumption 1 is not very restrictive. Notice that this assumption implies that the fitness functions are monotonically decreasing, which is a common characteristic in several scenarios, e.g., coordination games [4], biological models [12], congestion games [13], and so forth.

Next, we provide our main result on convergence of the $\epsilon$-dynamics.

Theorem 1: Convergence to $\epsilon$-equilibria. Let Assumption 1 hold, and let $E_{\epsilon}$ be a subset of int $\Delta$, where int $\Delta:=$ $\left\{x \in \mathbb{R}_{++}^{n}: \sum_{i=1}^{n} x_{i}=X\right\}$ is the interior of the simplex $\Delta$. The proposed $\epsilon$-dynamics (4) asymptotically converge to the set of $\epsilon$-equilibria $E_{\epsilon}$ of the underlying population game.

Proof: First, notice that since the set of $\epsilon$-equilibria belongs to the interior of the simplex $\Delta$ by assumption, this set is characterized by $E_{\epsilon}=\left\{x \in \Delta: \max _{i} f_{i}\left(x_{i}\right)-\right.$ $\left.\min _{i} f_{i}\left(x_{i}\right) \leq \epsilon\right\}$. To study the stability properties of $E_{\epsilon}$, let us consider the following Lyapunov function candidate

$$
V(x)=\max _{i \in \mathcal{S}} f_{i}\left(x_{i}\right)-\min _{i \in \mathcal{S}} f_{i}\left(x_{i}\right) .
$$

Clearly, $V(x) \geq 0$, for all $x \in \Delta$. Indeed, $V(x)=0$ only at the Nash equilibrium, i.e., at $x^{*} \in \Delta$ such that $f_{i}\left(x_{i}^{*}\right)=f_{j}\left(x_{j}^{*}\right)$, for all $i, j \in \mathcal{S}$. The derivative of (7) along the trajectories of the $\epsilon$-dynamics (4) is given by.

$$
\dot{V}(x)=\dot{V}_{M}(x)-\dot{V}_{m}(x),
$$

where $V_{M}(x):=\max _{i} f_{i}\left(x_{i}\right)$, and $V_{m}(x):=\min _{i} f_{i}\left(x_{i}\right)$.

Let us define the sets $\Omega_{M}=\left\{i: f_{i}\left(x_{i}\right)=V_{M}(x)\right\}$, and $\Omega_{m}=\left\{i: f_{i}\left(x_{i}\right)=V_{m}(x)\right\}$. Using the Clarke's generalized gradient [14], notice that $\dot{V}_{M}(x)$ and $\dot{V}_{m}(x)$ are given by

$$
\begin{aligned}
& \dot{V}_{M}(x)=\left\{\sum_{i \in \Omega_{M}} \lambda_{i} \frac{d f_{i}}{d x_{i}}\left(x_{i}\right) \dot{x}_{i}: \lambda_{i} \in \mathbb{R}_{+}, \sum_{i \in \Omega_{M}} \lambda_{i}=1\right\}, \\
& \dot{V}_{m}(x)=\left\{\sum_{i \in \Omega_{m}} \lambda_{i} \frac{d f_{i}}{d x_{i}}\left(x_{i}\right) \dot{x}_{i}: \lambda_{i} \in \mathbb{R}_{+}, \sum_{i \in \Omega_{m}} \lambda_{i}=1\right\} .
\end{aligned}
$$

If $i \in \Omega_{M}$ and $x \notin E_{\epsilon}$, then $\frac{d f_{i}}{d x_{i}}\left(x_{i}\right) \dot{x}_{i}=$ $\frac{d f_{i}}{d x_{i}}\left(x_{i}\right) \sum_{j \in \mathcal{S}} x_{j}\left[f_{i}(x)-\left(f_{j}(x)+\epsilon\right)\right]_{+}<0$ (since $\frac{d f_{i}}{d x_{i}}\left(x_{i}\right)<0$ by assumption). Therefore, $\dot{V}_{M}<0$, for all $x \notin E_{\epsilon}$. Furthermore, if $i \in \Omega_{m}$ and $x \notin E_{\epsilon}$, then $\frac{d f_{i}}{d x_{i}}\left(x_{i}\right) \dot{x}_{i}=-\frac{d f_{i}}{d x_{i}} x_{i} \sum_{j \in \mathcal{S}}\left[f_{j}(x)-\left(f_{i}(x)+\epsilon\right)\right]_{+}>0$. Hence, $\dot{V}_{m}>0$, for all $x \notin E_{\epsilon}$. Thus, $\dot{V}(x)<0$, for all $i \notin E_{\epsilon}$. Additionally, notice that $E_{\epsilon}$ is invariant under the $\epsilon$-dynamics (cf. Proposition 2) and $\dot{V}(x)=0$, for all $x \in E_{\epsilon}$. Therefore, according to the LaSalle's invariance principle [15], we can conclude that every solution of (4) converges to $E_{\epsilon}$ as $t \rightarrow+\infty$.

\section{Distributed EPSILON-DYNAMICS}

The $\epsilon$-dynamics described in (4) require full information to evolve. This fact is because they are derived from the meandynamics for well-mixed populations (2). In a large number of scenarios, full information dependency is undesirable (due to, for instance, privacy issues, scale of systems, limitations on the communication infrastructure, etc.). Therefore, we use the procedure proposed in [5] to relax the full information dependency of the $\epsilon$-dynamics. The core of the framework developed in [5] lies on the mean dynamics for non-wellmixed populations. Since the structure of a non-well-mixed population can be described by an undirected graph $\mathcal{G}=$ $\{\mathcal{S}, \mathcal{E}\}$, its mean dynamics are given by

$$
\dot{x}_{i}=\sum_{j \in \mathcal{N}_{i}} x_{j} \rho_{j i}(f(x), x)-x_{i} \sum_{j \in \mathcal{N}_{i}} \rho_{i j}(f(x), x), \forall i \in \mathcal{S},
$$

where $\mathcal{N}_{i}$ is the set of strategies that can interact with the $i^{\text {th }}$ strategy following the graph $\mathcal{G}$, i.e., $\mathcal{N}_{i}=\{j \in \mathcal{S}:(i, j) \in$ $\mathcal{E}\}$. Replacing the revision protocol (3) in the mean-dynamics for non-well-mixed populations (10), we obtain a version of the $\epsilon$-dynamics that only uses local information to evolve. These distributed $\epsilon$-dynamics are described as follows,

$$
\begin{aligned}
\dot{x}_{i}= & \sum_{j \in \mathcal{N}_{i}} x_{j}\left[f_{i}(x)-\left(f_{j}(x)+\epsilon\right)\right]_{+} \\
& -x_{i} \sum_{j \in \mathcal{N}_{i}}\left[f_{j}(x)-\left(f_{i}(x)+\epsilon\right)\right]_{+}, \forall i \in \mathcal{S} .
\end{aligned}
$$

Let us show that, although the distributed $\epsilon$-dynamics do not preserve all the appealing properties of (4), they still exhibit a desirable behavior.

\section{A. Simplex Invariance}

Distributed $\epsilon$-dynamics satisfies simplex invariance, as it is stated in the following proposition.

Proposition 3: Simplex invariance. Assume that the initial population state $x(0) \in \Delta$. If $x(t)$ evolves according to the distributed $\epsilon$-dynamics (11), then $x(t) \in \Delta$, for all $t \geq 0$.

Proof: Let $\mathcal{A}=\left[a_{i j}\right]$ be the adjacency matrix related to the graph $\mathcal{G}=\{\mathcal{S}, \mathcal{E}\}$, i.e., $a_{i j}=1$ if $(i, j) \in \mathcal{E}$, and $a_{i j}=0$ if $(i, j) \notin \mathcal{E}$. We recall that only undirected graphs are considered. Therefore, $a_{i j}=a_{j i}$.

1. Mass conservation: Since $\sum_{i \in \mathcal{S}} x_{i}(0)=X$ by assumption, it is sufficient to show that the quantity $\sum_{i \in \mathcal{S}} x_{i}(t)$ is positively invariant under (11). Notice that $\sum_{i \in \mathcal{S}} \dot{x}_{i}=\sum_{j \in \mathcal{S}} \sum_{i \in \mathcal{S}} a_{i j} x_{j}\left[f_{i}(x)-\left(f_{j}(x)+\epsilon\right)\right]_{+}-$ $\sum_{i \in \mathcal{S}} \sum_{j \in \mathcal{S}} a_{j i} x_{i}\left[f_{j}(x)-\left(f_{i}(x)+\epsilon\right)\right]_{+}$. Since $a_{i j}=a_{j i}$, the right-hand side of the latter equation can be rewritten as $\sum_{i \in \mathcal{S}} \dot{x}_{i}=\sum_{j \in \mathcal{S}} \sum_{i \in \mathcal{S}} a_{i j} x_{j}\left[f_{i}(x)-\left(f_{j}(x)+\epsilon\right)\right]_{+}-$ $\sum_{j \in \mathcal{S}} \sum_{i \in \mathcal{S}} a_{i j} x_{j}\left[f_{i}(x)-\left(f_{j}(x)+\epsilon\right)\right]_{+}$. This implies that $\sum_{i \in \mathcal{S}} \dot{x}_{i}=0$. Thus, $\sum_{i \in \mathcal{S}} x_{i}(t)$ is positively invariant. In conclusion, $\sum_{i \in \mathcal{S}} x_{i}(t)=X$, for all $t \geq 0$.

2. Nonnegativeness: To prove that $x_{i}(t) \geq 0$, for all $i \in \mathcal{S}$, and for all $t \geq 0$. We can employ the same arguments used in the proof of Proposition 1.

Notice that the statement of Proposition 3 does not impose special constraints on the graph $\mathcal{G}$. Therefore, simplex invariance is guaranteed under any population structure that can be described by an undirected graph. 


\section{B. Convergence Analysis}

Given a population structure described by the undirected graph $\mathcal{G}=\{\mathcal{S}, \mathcal{E}\}$, the equilibrium points $x^{*}$ of the distributed $\epsilon$-dynamics are characterized by the following set,

$$
\hat{E}_{\epsilon}=\left\{x^{*} \in \Delta: x_{i}^{*}>0 \Rightarrow f_{i}\left(x_{i}^{*}\right) \geq f_{j}\left(x_{i}^{*}\right)-\epsilon, \forall j \in \mathcal{N}_{i}\right\} .
$$

Therefore, although any $\epsilon$-equilibrium is a rest point of the distributed $\epsilon$-dynamics, a rest point of these dynamics is not necessarily an $\epsilon$-equilibrium (unless $\mathcal{N}_{i}=\mathcal{S}$, for all $i \in \mathcal{S}$, i.e, unless the graph is complete). This is the main difference of the distributed $\epsilon$-dynamics compared to the $\epsilon$-dynamics that use full information (4).

If $\mathcal{G}$ is connected, then we can make a better characterization of the equilibrium set $\hat{E}_{\epsilon}$. To do this, let $d(i, j)$ be the distance between nodes $i$ and $j$ of $\mathcal{G}$ (the distance between two nodes is equal to the number of edges in a shortest path that connects these nodes). Moreover, let $D$ be the diameter of the graph $\mathcal{G}$, which is defined as $D=\max _{i, j \in \mathcal{S}} d(i, j)$. For connected graphs, it can be shown that $\hat{E}_{\epsilon} \subseteq E_{D \epsilon}$, where $E_{D \epsilon}$ is the set of $D \epsilon$-equilibria of the population game, i.e.,

$E_{D \epsilon}=\left\{x \in \Delta: x_{i}>0 \Rightarrow f_{i}(x) \geq f_{j}(x)-D \epsilon, \forall i, j \in \mathcal{S}\right\}$

Next, we provide a convergence result for the distributed $\epsilon$-dynamics.

Proposition 4: Convergence. Let Assumption 1 hold, and let $\hat{E}_{\epsilon}$ be a subset of int $\Delta$. If the undirected graph $\mathcal{G}$ that describes the population structure is connected, then the distributed $\epsilon$-dynamics asymptotically converge to the set $\hat{E}_{\epsilon} \subseteq E_{D_{\epsilon}}$.

Proof: In order to study the stability properties of $\hat{E}_{\epsilon}$, we use the Lyapunov function candidate $V(x)$ defined in (7), and employ the same procedure described in the proof of Theorem 1 to obtain that $\dot{V}(x)=\dot{V}_{M}(x)-\dot{V}_{m}(x)$, where $\dot{V}_{M}(x)$ and $\dot{V}_{m}(x)$ are given in (8) and (9), respectively.

Notice that, if $i \in \Omega_{M}$, then $\frac{d f_{i}}{d x_{i}}\left(x_{i}\right) \dot{x}_{i}=$ $\frac{d f_{i}}{d x_{i}}\left(x_{i}\right) \sum_{j \in \mathcal{N}_{i}} x_{j}\left[f_{i}(x)-\left(f_{j}(x)+\epsilon\right)\right]_{+} \leq 0$. Therefore, $\dot{V}_{M} \leq 0$. Furthermore, if $i \in \Omega_{m}$, then $\frac{d f_{i}}{d x_{i}}\left(x_{i}\right) \dot{x}_{i}=$ $-\frac{d f_{i}}{d x_{i}} x_{i} \sum_{j \in \mathcal{N}_{i}}\left[f_{j}(x)-\left(f_{i}(x)+\epsilon\right)\right]_{+} \geq 0$. Hence, $\dot{V}_{m} \geq 0$. Thus, $\dot{V}(x) \leq 0$. Additionally, since $\mathcal{G}$ is connected by assumption, $\dot{V}(x)=0$ only if $x \in \hat{E}_{\epsilon}$. Therefore, we can use the LaSalle's invariance principle [15] to conclude that every solution of (11) converges to $\hat{E}_{\epsilon}$ as $t \rightarrow+\infty$.

Under the conditions given in the statement of Proposition 4, a simpler characterization of the set $E_{D \epsilon}$ is as follows,

$$
E_{D \epsilon}=\left\{x \in \Delta: \max _{i \in \mathcal{S}} f_{i}(x)-\min _{i \in \mathcal{S}} f_{i}(x) \leq D \epsilon\right\}
$$

In the following sections, we illustrate the applicability of the proposed $\epsilon$-dynamics in some engineering problems.

\section{Vi. Control with Noisy Measurements}

Figure 1 shows a case study composed by $m$ tanks. The control objective is to maintain all the tank levels at the same reference $r$, while a physical constraint given by a limited amount of water flow $X$ must be satisfied. Moreover, we

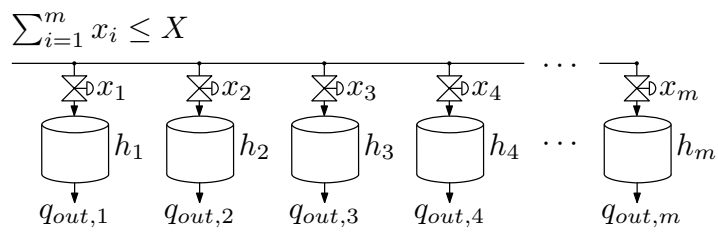

Fig. 1. Case study composed by $m$-tanks.

consider that there is an additive noise in the measurements of each tank level. This noise is denoted by $d_{i} \in\left[\begin{array}{ll}-\underline{d} & \bar{d}\end{array}\right]$.

The dynamics of the $i^{t h}$ tank are given by $\dot{h}_{i}=x_{i}-K_{i} h_{i}$, where $h_{i}$ is the water level, $x_{i}$ is the inflow, and $K_{i}$ is a constant factor characterizing the outflow.

We compare the performance of the closed-loop system when the tanks inflows are controlled via the Smith dynamics (i.e., the $\epsilon$-dynamics with $\epsilon=0$ ) and the $\epsilon$-dynamics with $\epsilon>0$. This is done by taking as strategies the tanks, and as population mass the available water flow $X$. Hence, $x_{i}$ (i.e., the water inflow allocated to the $i^{\text {th }}$ tank) corresponds to the proportion of the players mass that choose the $i^{t h}$ strategy. We propose a case study composed by $m=10$ tanks, with a constant available water flow given by $X=25$, and noise for each measurement in the range $[-0.5,0.5]$. We assume that there is a local controller per valve, which guarantees that each inflow reaches the reference $x_{i}$ imposed by the population dynamics. The limited available water flow establishes a constraint over all the inflows. This constraint is managed by adding a nonnegative slack variable $x_{m+1}$, i.e., we add an strategy $(m+1)$, and obtain according to the simplex invariance that $\sum_{i=1}^{m+1} x_{i}=X$ (cf. Proposition 1). Thus, $\sum_{i=1}^{m} x_{i} \leq X$.

The fitness function associated with the $i^{t h}$ tank is selected as the level error, i.e., $f_{i}=r-h_{i}^{m}$, where $h_{i}^{m}=h_{i}+d_{i}$ is the measured level. Thus, more water inflow is allocated to those tanks with higher error. Figure 2(a) shows the evolution of the tanks levels controlled via the Smith dynamics. Notice that the disturbances in the measurements affect the values of the fitness functions. Consequently, the performance of this controller exhibits permanent oscillations that could have a negative effect on the systems actuators.

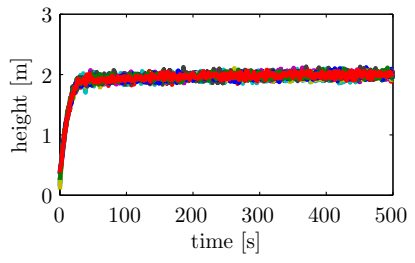

(a)

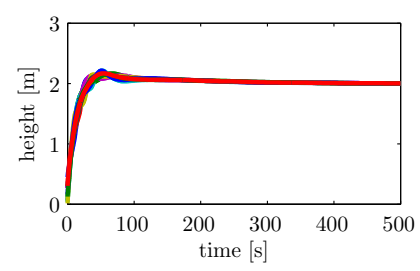

(b)
Fig. 2. Evolution of the tanks levels under: (a) Smith dynamics $(\epsilon=0)$ and (b) $\epsilon$-dynamics $(\epsilon=0.5)$.

In order to mitigate the problem caused by the noisy measurements, we implement the $\epsilon$-dynamics. The noise included in the measurements is neglected as long as the value of $\epsilon \geq \bar{d}$. This is because the possible increment of the fitness functions do not overtake the threshold that makes the 
players (water inflow) want to change them strategies (tanks) when they are in an $\epsilon$-equilibrium. Therefore, the distribution of population mass among the strategies (i.e., allocation of the water flow among the tanks) remains constant. Figure 2(b) shows the performance of the $\epsilon$-dynamics with $\epsilon=0.5$. It can be seen the significant improvement and the mitigation of the noise under these dynamics.

\section{Community Wealth Distribution}

Consider a community composed by $n$ individuals. Let $x_{i}>0$ be the wealth of the $i^{t h}$ individual. Moreover, assume that the $i^{\text {th }}$ individual can only interact with a set of individuals in the community (its neighbors). These "allowed" interactions are described by an undirected graph $\mathcal{G}=\{\mathcal{V}, \mathcal{E}\}$, where: $\mathcal{V}$ is the set of nodes, which are related to the individuals of the community, i.e., $\mathcal{V}=\{1, \ldots, n\}$; and $\mathcal{E}$ is the set of edges, which are related to the interaction links between individuals, i.e., if $(i, j) \in \mathcal{E}$, then the $i^{\text {th }}$ individual can interact with the $j^{\text {th }}$ individual. Our objective is to design a wealth distribution policy that mitigates the wealth gap in the considered community.

Previous works have employed classic consensus protocols [8]. However, the scenario in which all the individuals achieve the same wealth is not realistic. Hence, instead of using consensus algorithms, we propose to apply the distributed $\epsilon$-dynamics with $f_{i}\left(x_{i}\right)=-x_{i}$, for all $i \in \mathcal{S}$. Notice that this is equivalent to use the revision protocol $\rho_{i j}=\left[x_{i}-\left(x_{j}+\epsilon\right)\right]_{+}$, i.e., the $i^{t h}$ individual only donates part of his/her wealth to the $j^{\text {th }}$ individual if the wealth gap between these two people is greater than a threshold $\epsilon>0$.

In order to illustrate the performance of the proposed method, let us consider a community composed by 100 individuals, where interactions among individuals are described by a path graph. Initially, the wealths of individuals are distributed as follows: $x_{1}(0)=\$ 0.5, x_{2}(0)=\$ 1, x_{3}(0)=$ $\$ 1.5, \ldots, x_{99}(0)=\$ 49.5, x_{100}=\$ 50$ (i.e., $x_{i}(0)=\$ 0.5 i$ ). Figure 3(a) shows the evolution of the wealth of each individual if the community applies a wealth distribution policy based on the $\epsilon$-dynamics (11) with $\epsilon=\$ 0.02$. Notice that the wealth gap decreases along the time and the wealth distribution reaches an equilibrium $x^{*}$ that belongs to the set $E_{D \epsilon}$ (cf. Equation (13)), where $D=100$. This fact is because $\max _{i \in \mathcal{S}} x_{i}^{*}-\min _{i \in \mathcal{S}} x_{i}^{*}<\$ 2$, i.e., the wealth gap decreases to a value that is lower than $D \epsilon$. On the other hand, Figure 3(b) shows the evolution of the individuals' wealth under the same scenario described above, but using a complete graph, i.e., individuals have full information. In this case, evolution of the wealth distribution is faster, and the wealth gap decreases to a value equal to $\epsilon$, i.e., an $\epsilon$ equilibrium is reached.

\section{CONCLUSIONS}

We have deduced a centralized and distributed class of population dynamics parameterized by a scalar term $\epsilon$ as an extension of the classic pairwise comparison revision protocol and the mean dynamics. As a result, the $\epsilon$-dynamics have been obtained, which converge to $\epsilon$-equilibria (a relaxed

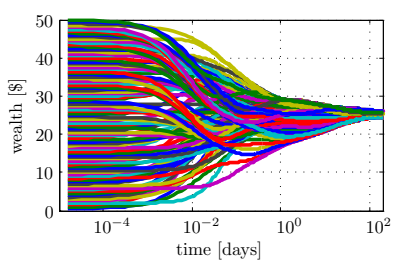

(a)



(b)
Fig. 3. Evolution of the wealth of each individual under: (a) Distributed $\epsilon$ dynamics $(\epsilon=0.02)$, and (b) $\epsilon$-dynamics with full information $(\epsilon=0.02)$

condition of Nash equilibria). Stability of the $\epsilon$-equilibrium and invariance of the set of allowed population states (simplex) have been formally proven. Furthermore, a case study with imperfect information (a more realistic scenario than those scenarios considered in other related works) has shown the enhancement of the closed-loop performance with an $\epsilon$ dynamics-based controller, which is capable to mitigate noisy measurements in comparison to other population-dynamicsbased controllers that converge to a Nash equilibrium. Besides, we have taken advantage of the properties of the $\epsilon$ dynamics to develop a model of wealth distribution in poor communities.

\section{ACKNOWLEDGMENTS}

The authors would like to thank Professor K. M. Passino for his valuable inputs.

\section{REFERENCES}

[1] I. Menache and A. Ozdaglar, Network games: Theory, models, and dynamics, vol. 4. Morgan \& Claypool Publishers, 2011.

[2] C. Daskalakis, P. Goldberg, and C. Papadimitriou, "The complexity of computing a Nash equilibrium," pp. 71-78, ACM Press, 2006.

[3] H. Bosse, J. Byrka, and E. Markakis, "New algorithms for approximate nash equilibria in bimatrix games," Theoretical Computer Science, vol. 411, pp. 164-173, 2010.

[4] W. H. Sandholm, Population games and evolutionary dynamics. Cambridge, Mass. MIT Press, 2010.

[5] J. Barreiro-Gomez, G. Obando, and N. Quijano, "Distributed population dynamics: Optimization and control applications," IEEE Transactions on Systems, Man, and Cybernetics: Systems. DOI: 10.1109/TSMC.2016.2523934, 2016.

[6] E. Ramirez-Llanos and N. Quijano, "A population dynamics approach for the water distribution problem," International Journal of Control, vol. 83, no. 9, pp. 1947-1964, 2010.

[7] G. Obando, A. Pantoja, and N. Quijano, "Building Temperature Control based on Population Dynamics," IEEE Transactions on Control Systems Technology., vol. 22, no. 1, pp. 404-412, 2014.

[8] K. Passino, Humanitarian engineering. Creating technologies that help people. Bede Publishing, Columbus, Ohio, 2nd ed., 2015.

[9] J. I. Poveda and N. Quijano, "Shahshahani gradient-like extremum seeking," Automatica, vol. 58, pp. 51-59, 2015.

[10] J. Barreiro-Gomez, N. Quijano, and C. Ocampo-Martinez, "Constrained distributed optimization: A population dynamics approach," Automatica. DOI: 10.1016/j.automatica.2016.02.004, 2016.

[11] A. Pantoja and N. Quijano, "A population dynamics approach for the dispatch of distributed generators," IEEE Transactions on Industrial Electronics, vol. 58, pp. 4559-4567, 2011.

[12] N. Quijano and K. M. Passino, "The ideal free distribution: theory and engineering application,' IEEE Transactions on Systems, Man, and Cybernetics, Part B: Cybernetics, vol. 37, no. 1, pp. 154-165, 2007.

[13] H. Tembine, E. Altman, R. El-Azouzi, and Y. Hayel, "Evolutionary games in wireless networks," IEEE Transactions on Systems, Man, and Cybernetics, Part B: Cybernetics, vol. 40, no. 3, pp. 634-646, 2010.

[14] F. Clarke, Optimization and nonsmooth analysis, vol. 5. Siam, 1990.

[15] H. K. Khalil, Nonlinear Systems. Upper Saddle River, NJ: Prentice Hall, 2002. 